\title{
Seelische Belastung: Was bringt die Aushändigung eines Tumornachsorgeplans?
}

\begin{abstract}
Gerade in den ersten fünf Jahren nach einer Krebsdiagnose ist die psychische Belastung der Patienten groß - mit möglichen Sekundärfolgen wie Depression, Angst oder posttraumatischem Stresssyndrom. Zur strukturierten Tumornachsorge erhalten Patienten in den USA einen Nachsorgeplan und eine Therapiezusammenfassung. Sind diese hilfreich für den Patienten?
\end{abstract}

" In den USA erhalten Erwachsene nach Ende der Tumortherapie seit einigen Jahren einen „survivorship care plan" (SCP) mit Nachsorgeplan (FCI; "follow-up care instructions") und Therapiezusammenfassung (TS; „treatment summary"). In früheren Studien wurde gezeigt, dass diese Informationen wirklich zu konsequenterer Nachsorge und geringerem psychischem Stress führen. Nun wurde untersucht, ob sie tatsächlich Einfluss, insbesondere auf das Auftreten depressiver Symptome, haben.

Dazu analysierten die Forscher eine repräsentative Stichprobe überlebender Tumorpatienten, die mehr als ein Jahr nach der Diagnose per Telefon an dem bevölkerungsweiten 2010-BRFSS (Be- havioral Risk Factor Surveillance System)-Survey teilnahmen. In einer Stichprobe aus sechs Bundessstaaten wurden hier die optionalen Module "Anxiety and Depression“ und "Cancer Survivorship“ berücksichtigt. Depressive Symptome wurden mittels PHQ8 (Patient Health Questionnaire, 8 Items) erfasst. Um zu prüfen, ob FCI und TS (einzeln und in Kombination) mit Depressionssymptomen assoziiert sind, wurden die 3.191 Patienten in zwei Gruppen unterteilt: die „Kurzzeitüberlebenden" (Krebsdiagnose vor $\leq 5$ Jahren; 32,8\%) und die „Langzeitüberlebenden“" (Krebsdiagnose vor $>5$ Jahren; $67,2 \%)$.

Bei Kurzzeitüberlebenden spielt vor allem der FCI eine Rolle. So war der
Faktor keinen FCI erhalten zu haben bei ihnen signifikant mit einem Anstieg der Depressionssymptome assoziiert (adjustierte Odds Ratio [AOR] 2,27, 95\%-Konfidenzintervall [95\%-KI] 1,15-4,45). Bei den Langzeitüberlebenden kommt dagegen der TS größere Bedeutung zu. Bei ihnen war der Faktor keine TS erhalten zu haben signifikant mit einem Anstieg der Depressionssymptome assoziiert (AOR 1,86, 95 \%-KI 1,05-3,29).

Fazit: Überlebende Tumorpatienten sollten strukturierte Anleitungen zur Nachsorge und Therapiezusammenfassungen erhalten. Sie können kurzund langfristig davon profitieren, was sich an einer deutlichen Verringerung depressiver Symptome zeigt. $\quad(B r S)$

Quelle:

Oancea SC, Cheruvu VK: Psychological distress among adult cancer survivors: importance of survivorship care plan. Support Care Cancer. 2016;24(11):4523-31.

\section{Eine allgemeine Thromboseprophylaxe für Krebspatienten mit ambulanter Chemotherapie wird derzeit nicht empfohlen. In einem Cochrane-Review wurde nun die Datenlage zu Nutzen und Risiken dieser Maßnahme gesichtet.}

\begin{abstract}
") Das bei Patienten mit aktiver Krebserkrankung ohnehin erhöhte Risiko für venöse Thromboembolien (VTE) wird durch eine Chemotherapie zusätzlich gesteigert. Ob die Patienten von einer Thromboseprophylaxe profitieren, ist jedoch unklar. Neben dem Thromboserisiko ist die erhöhte Blutungsgefahr zu beachten, die Tumor und Chemotherapie verursachen können.
\end{abstract}

Ärzte der Cochrane-Gefäßgruppe haben deswegen erneut in einem Review geprüft, was eine Thrombose- prophylaxe bei ambulanten Patienten mit Chemotherapie bewirkt. Ihre Schlussfolgerungen sind die gleichen wie nach dem ersten Review im Jahr 2012: Niedermolekulare Heparine (NMH) führen zu einer signifikanten Reduktion von symptomatischen VTE. Wie sie sich auf das Auftreten von schweren Blutungen auswirken, lässt sich nicht definitiv beurteilen. Eine routinemäßige VTE-Prophylaxe für ambulante Patienten kann daher noch nicht empfohlen werden.

\section{Weniger VTE, nicht signifikant mehr schwere Blutungen}

In der Analyse wurden 26 randomisierte kontrollierte Studien mit mehr als 12.000 Patienten berücksichtigt. Alle VTE-Präventionsmaßnahmen waren medikamentöser Art. Die meisten Patienten litten an lokal fortgeschrittenem oder metastasiertem Krebs.

NMH wurden in 18 Studien untersucht. Im Vergleich zu Patienten ohne Prophylaxe war das Risiko für symptomatische VTE um $46 \%$ und für symptomatische Lungenembolien um $41 \%$ reduziert. Die Risikosenkung war unabhängig von der Art des verwendeten NMH oder der Krebserkrankung. Das 